BULLETIN OF THE

AMERICAN MATHEMATICAL SOCIETY

Volume 80, Number 5, September 1974

\title{
REPRESENTATION OF PARTIALLY ORDERED LINEAR ALGEBRAS
}

\author{
BY RICHARD C. METZLER
}

Communicated by Robert Bartle, December 12, 1973

In [2] and [3] a condition on partially ordered linear algebras (pola's) is defined, and it is shown that Dedekind $\sigma$-complete polas satisfying this condition have many of the properties of function spaces. Using a theorem of $\mathrm{H}$. Nakano we can show, even without the hypothesis that the pola is Dedekind $\sigma$-complete, that any such pola is isomorphic to a pola of continuous, almost-finite, extended-real-valued functions. If $A$ is a pola with multiplicative identity 1 the condition mentioned is:

$\mathrm{P}_{1}$. If $x \in A$ and $x \geqq 1$, then $x$ has an inverse and $x^{-1} \geqq 0$.

THEOREM. In order for an Archimedean pola $A$ with identity 1 to be isomorphic to a pola of continuous, almost-finite, extended-real-valued functions on a compact Hausdorff space $X$, it is sufficient that $\mathbf{P}_{1}$ hold for $A$. The condition is necessary also if $A_{1}=\left\{y \in A\right.$ : there exists $\alpha \in R^{+}$with $-\alpha 1 \leqq y \leqq \alpha 1\}$ is complete in the order unit norm derived from 1 and if the image of $A_{1}$ separates points in $X$.

Proof. The standard completion procedure for Archimedean ordered linear spaces shows that $A$ is isomorphic with an order dense subspace $\hat{A}$ of a Dedekind complete linear lattice $D$. In $[4$, p. 150] it is shown that the multiplication on $\hat{A}$ can be extended to $D$ in such a way that $D$ is a pola if the following continuity condition is satisfied: For every subset $B$ of $A, \inf B=0$ implies $\inf (a B)=\inf (B a)=0$ for all positive elements $a$ in $A$. Given $\mathrm{P}_{1}$, multiplication by $(a+1)^{-1}$ shows this condition is satisfied. Thus $D$ is a linear lattice pola and the order density of $\hat{A}$ shows (since 1 is easily seen to be a weak order unit for $A$ ) that the image of 1 is a weak order unit for $D$. Now $D$ (and hence $A$ ) has a representation of the type desired by [1, Corollary, p. 625].

To prove the second statement we note that the assumptions, together with the Stone-Weierstrass theorem, give the result that if $A \rightarrow \hat{A}$ is the isomorphism then $\hat{A}_{1}=C(X)$. Then, given any $x$ in $A$ such that $x \geqq 1$,

AMS (MOS) subject classifications (1970). Primary 06A70; Secondary 06A65.

Key words and phrases. Partially ordered linear algebra, representation by functions.

Copyright (c) American Mathematical Society 1974 
we can define an $f$ in $C(X)$ by $f(t)=1 / \hat{x}(t)$ for all $t$ in $X$ (with $1 / \infty$ set equal to 0$)$. Then there exists $z$ in $A_{1}$ such that $\hat{z}=f$ and it is clear that $z=x^{-1}$ and $z \geqq 0$.

Note that it is not enough to know that $A$ separates points of $X$ to conclude that $A_{1}$ does. This shows the need for the separation assumption. Also, it is easy to see that if $A$ is Dedekind $\sigma$-complete, then $A_{1}$ is complete in the order unit norm, so this case is included.

An immediate consequence of this theorem is the useful result that if a pola is Archimedean, has an identity, and satisfies $\mathbf{P}_{\mathbf{1}}$, then it is necessarily commutative.

\section{REFERENCES}

1. S. J. Bernau, Unique representation of Archimedean lattice groups and normal Archimedean lattice rings, Proc. London Math. Soc. (3) 15 (1965), 599-631; Addendum: ibid. (3) 16 (1966), 384. MR 32 \#144; \#7652.

2. T. Dai, On a special class of partially ordered linear algebras, J. Math. Anal. Appl. 40 (1972), 649-682.

3. R. E. DeMarr, A class of partially ordered linear algebras, Proc. Amer. Math. Soc. 39 (1973), 255-260.

4. H. Nakano, Modern spectral theory, Maruzen, Tokyo, 1950. MR 12, 419.

Department of Mathematics, University of New Mexico, Albuquerque, New MeXICo 87106 